\title{
Supporting the life cycle of complex technical systems on the basis of intelligent technologies and predictive analytics
}

\author{
V N Blinov ${ }^{1}$, S S Valeev ${ }^{2}$, N V Kondratyeva ${ }^{2}$, R R Karimov ${ }^{2}$, A S Kovtunenko ${ }^{2}$ \\ and $\mathrm{E}$ A Kuzmina ${ }^{2}$ \\ ${ }^{1}$ Omsk State Technical University, Pr. Mira Str., 11, Omsk, Russia, 644050 \\ ${ }^{2}$ Ufa State Aviation Technical University, K. Marx Str., 12, Ufa, Russia, 450008 \\ e-mail: vss2000@mail.ru
}

\begin{abstract}
The application of predictive analytics in the design, production and operation to achieve the efficiency of the life cycle of complex technical systems is discussed. A predictive model of information support for the life cycle of a microsatellite propulsion system based on a neural network system is proposed. The predictive model can solve the problem of estimating fuel consumption, diagnosing and detecting possible failures of a small propulsion system.
\end{abstract}

\section{Introduction}

The modern systems of information support of the life cycle of the large scale technical objects, such as airplanes, gas turbine engines, are widely used. But for small dynamic objects, such as drones or microsatellites, the use of life-cycle information support systems is not so obvious for many reasons. One of the main reasons why researchers and manufacturers are not ready for applying this technology is lack of the experience and rather expensive life cycle information support systems equipment and software.

The microsatellite can be considered as a subclass of the cyberphysical system functioning in autonomous mode during its mission for rather a long period of time. It is possible to control its position and payload periodically remotely from ground flight center in telecommunication sessions. An operator can send control commands through, for example, GlobalStar, and change the operation mode for the microsatellite effective use with help of special software-hardware interface [1-8].

The mission tasks of microsatellite in information sense is the data collecting, processing, and transmitting to the ground flight center the compressed information about its environment and flying conditions. Microsatellite, as a subset of a cyber physicalsystem, contains a lot of mechatronics subsystems: micro electromechanical elements and mechanisms. So, it can be considered as a complex technological product, which is designed within the enterprises cooperation on the base of information flows interchanging $[9,10]$. On the stage of designing of the life cycle for autonomous technical objects, the designers have to keep in mind the possible uncertainty factors which can influence on the object operation and can cause failures of onboard equipment, energy overconsumption, noise influence and the other different factors. So, to provide the efficiency of the microsatellite mission, the information support based on predictive analytics technologies can be applied $[11,12]$.

\section{The life cycle of the complex technical system}

The small size mobile observing systems are very urgent nowadays in the most of scientific and commercial applications. One of the ways to achieve the life cycle efficiency for such a system is intelligent information support on the stages of design, production and exploitation. 
A low-weight mobile autonomous system exploited without any human service during long-term periods is considered as a complex technical system. The human operator can control the complex technical system remotely in short sessions. For this purpose, the operator sends commands and sets temporal programs for complex technical system exploitation with aid of software-hardware interface. The main aims of a complex technical system are collecting, processing and transmitting objective information about its environment and also transportation, mechanical and other interactions with environmental objects.

The complex technical system contains a number of technical subsystems, microelectromechanical components and mechanisms, and software-hardware components. Therefore, a small size mobile observing system is a high technological product created within wide international cooperation.

The efficient exploitation of the complex technical system during its short active lifetime has to be assured. Increasing exploitation efficiency requires creating the integrated hardware-software system for the information support of the complex technical system life cycle.

One of the problems hard to solve is uncertainty caused by failures of onboard software-hardware subsystems, information distortion, external noise influence, energy losses, etc.

The complex technical system lifecycle includes six stages: system project, designing, manufacturing, implementation, operation and utilization. But only the operation stage assures profitable usage. For today, introductory stages duration (1-4) is approximately equal to the duration of exploitation stage (5). The introductory stages still remain rather expensive from the investor point of view. To advance overall efficiency, it is necessary to decrease duration of the stages 1-4 and increase the useful effect on the stage 5. The main stages of the complex technical system life cycle and benefits $P(t)$ and total costs $\mathrm{Z}(t)$ functions are shown in the figure 1 .

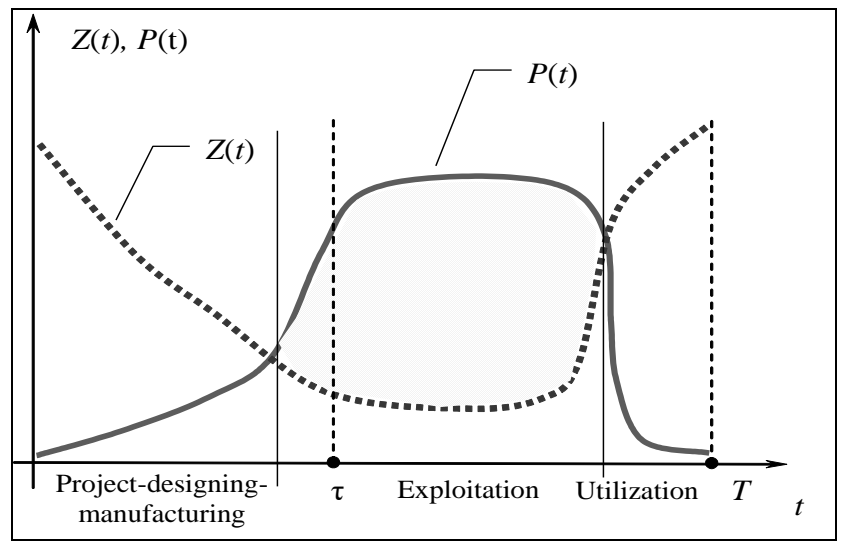

Figure 1. Benefits and total costs $Z(t)$ functions on the main stages of the complex technical system life cycle.

Target efficiency of the complex technical system life cycle could be estimated as the integral value on the base of "the through life cycle" mathematical models:

$$
E(t)=\int_{0}^{t}(P(t)-Z(t)) d t
$$

When planning and correcting life cycle operations and making exploitation programs, it is either required to estimate useful effects $E_{\tau}$ and make the forecast of probable negative or positive aftereffects $E_{\mathrm{T}-\tau}^{\mathrm{FC}}$ - for example, the complex technical system resource degradation. This allows choosing the optimal option not for an operation at temporal time $\tau$ only, but for the entire further life cycle $T$ :

$$
E_{\mathrm{LC}, i}=E_{\tau}+E_{\mathrm{T}-\tau}^{\mathrm{FC}}=\int_{0}^{\tau}(P(t)-Z(t)) d t+\int_{\tau}^{\mathrm{T}}(P(t)-Z(t)) d t
$$




$$
E_{\mathrm{LC}}=\max _{i}\left\{E_{\mathrm{LC}, i}\right\} .
$$

To obtain the optimal effect $E_{\mathrm{LC}}$, the following tasks of the information support of the complex technical system life cycle should be solved:

- the intelligent support of complex technical system at the design stage;

- keeping the information on the complex technical system condition the actual, operational and authentic;

- estimating and forecasting the complex technical system condition;

- the decision making support using "through the life cycle" intelligent models to estimate target efficiency on the life cycle stages;

- simulation of "optimum" and "worst-case" situations while the life cycle participants interacting on the base of decision trees;

- the diagnostic and decision-making on the base of mathematical and intelligent models of the complex technical system onboard systems.

All these measures will promote total efficiency of the complex technical system life cycle due to the effective interactions between participants, high awareness of the life cycle participants and decrease wrong decision probability during the life cycle stages.

\section{The life cycle of the complex technical object and predictive analytics}

In sense of the life cycle paradigm of the microsatellite, the amount of data generated on prototyping and testing stages are more times bigger then data had generated on the implementation stage (figure 2).

So it is obvious, if we are trying to optimize total expenditures for the life cycle of the microsatellite:

- It is necessary to decrease the durations of the stages from prototyping to implementation.

- It is needed to increase the usefulness of information that had been generated on before the implementation stage.

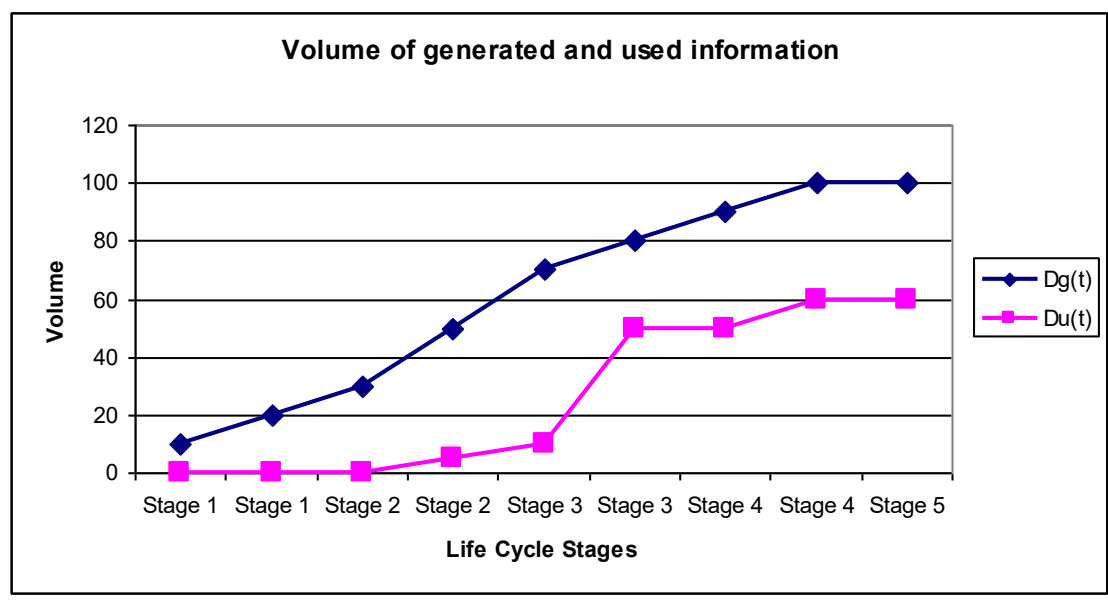

Figure 2. Volume of information generated on all stages of life cycle.

In general, the effectiveness of generated information $D_{E}$ on stages of the life cycle of complex technical object can be presented as the solution of optimization task:

$$
D_{E}(t)=\int_{0}^{t}\left(D_{G}(t)-D_{U}(t)\right) d t \rightarrow \min ,
$$

where $D_{G}$ is generated information, and $D_{U}$ is used information. 
The following tasks of the predictive analytics application have to be solved in order to obtain the positive effect in sense of (1):

- big data technologies can be applied on all stages of the life cycle. Supporting actual, operational and authentic information about the state of the complex technical object operation with help of estimating and forecasting the complex technical object state on the basis of predictive models;

- the decision making support using different predictive models to estimate the meaning of goal function on the different stages;

- simulation of different "suboptimum" and "worse" cases on the base of multi-agent real time simulation system;

- diagnostics on the base of on-line predictive models of complex technical object state with application of onboard computers.

These tasks can increase the economic efficiency of the life cycle of complex technical system due to the effective interactions of all information subsystems with application of predictive analytics on all stages of the life cycle.

\section{Intelligent technologies of the life cycle information support for the complex technical system}

Due to the essential uncertainty on the design, manufacturing and exploitation stages of the complex technical system life cycle, the intelligent technologies become rather actual for the life cycle information support.

First of all, on the stage of system design, developers have to solve the problem of optimal structure of the complex technical system. This problem is proposed to solve with genetic algorithms for optimizing information model of complex technical system.

The second problem is that on the exploitation stage human controller has to diagnose technical condition of the remote complex technical system, estimate internal complex technical system resources and provide process monitoring on the base of telemetry data stream.

The third problem is when planning system operations, it is necessary to build a simulation model to choose the optimal life cycle strategy and avoid worst-case situations. Last two problems are proposed to solve with help of the through-life cycle neural network model.

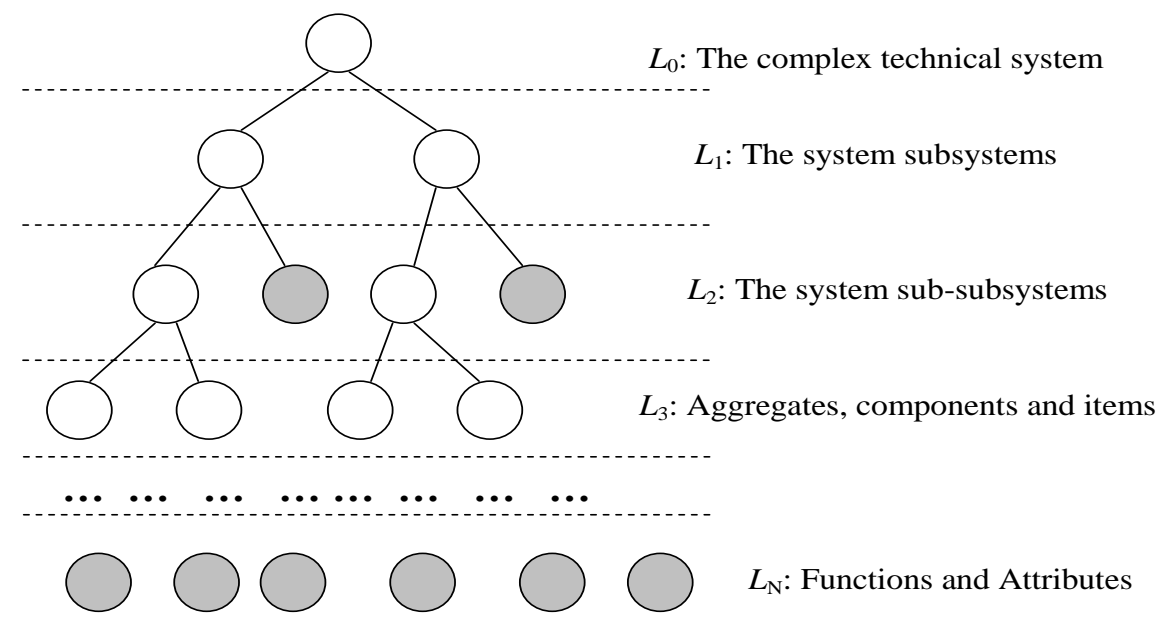

Functions, Attributes, Documents, Data Containers

Figure 3. The information hierarchical model of the complex technical system as a genetic individual.

The information model of the complex technical system has hierarchy multilevel structure which is represented as a tree graph, the root node of which is the complex technical system itself (figure 3 ). On the models' second level, the complex technical system subsystems are involved, on the next levels the complex technical system aggregates, components and items. The terminal nodes of the model are 
the characteristics, attributes of the complex technical system as well as complex information objects, such as documents, data containers and others. The terminal nodes are painted hatched in figure 3.

One multilevel structure presented in figure 3 describes an individual of evolution procedures. Middle nodes as subsystems and aggregates (for example, power supply, engine, cameras, etc.) are the chromosomes of the individual, and the terminal nodes - characteristics and attributes - are the genes.

The populations, obtained by genetic algorithms within crossover and mutation, allow producing amount of the different versions of the complex technical system. Then individuals are selected with the aid of chosen criteria - fitness function.

The transitions between PDM database and the individual hierarchy could be realized via SGML (XML) transformations.

\section{The Microsatellite Engine Predictive Model}

The research and practical implementation work that was carried out and the results of flight and ground tests of ammonia propulsion engine with a thrust price of up to $4 \mathrm{~W} / \mathrm{mN}$ for electrothermal micro engine of up to $30 \mathrm{mN}$ showed their high efficiency in the small satellites "Demonstrator", "Orbkomm" and "UgatuSat" [2].

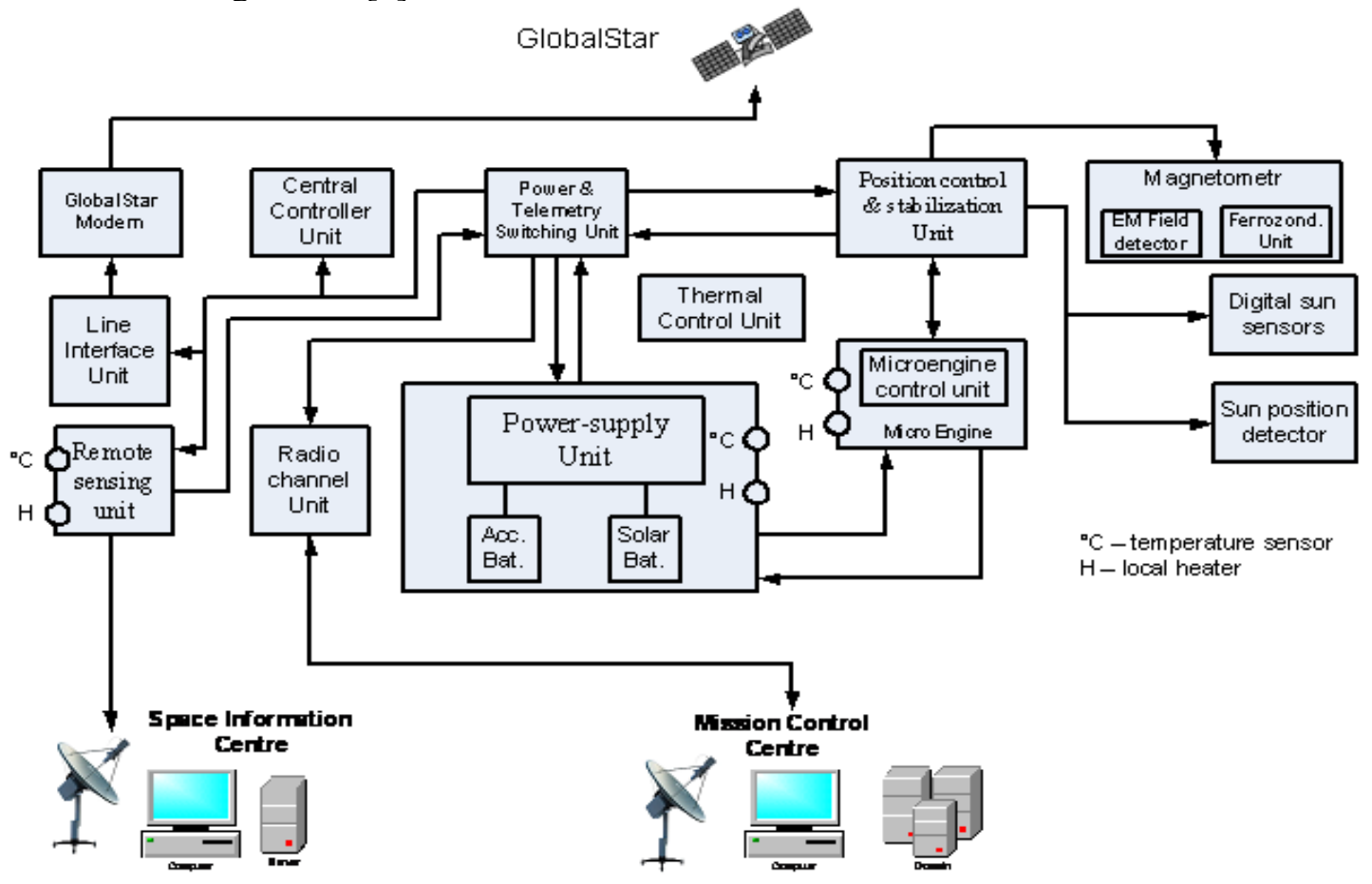

Figure 4. Predictive estimation of remaining fuel on the base of telemetry data.

Let us consider the resource and state assessment subsystem on the example of a propulsion system, which is designed to correct the orbital position of the micro satellite. The propulsion system consists of a cylindrical fuel tank, a filter, an electropneumatic valve, an evaporator for converting liquid ammonia into a gaseous state, a pressure regulator to maintain a predetermined pressure value, as well as an electrothermal engine. The considered PS is developed in the PE "Polet" (Omsk) and is used on Russian and foreign microsatellites.

The main non-renewable resource on board the microsatellite is the fuel supply. The task of determining the amount of fuel on board the spacecraft is very relevant due to the lack of a fuel sensor, extreme external conditions, the inability to refuel and other services. The factors of uncertainty include the occurrence of failures of the components of the control, loss of fuel due to leaks, chemical decomposition of fuel, etc. 
The principal pneumohydraulic scheme of propulsion system is presented in figure 5, which includes an electrothermal engine, pressure sensor, pressure regulator (controller), evaporator, electropneumatic valve, filter, ammonia tank (fuel tank).

The key feature of the considered propulsion system is the formation of small impulses of thrust and operation in the "cold start" mode, as a result of which the thrust and fuel consumption are functions of time.

The principle of operation of a micro jet engine with an ammonia electro thermal engine is based on the dissociation of ammonia with its decomposition into hydrogen and nitrogen. Due to the decomposition and, accordingly, a twofold decrease in the molecular weight of the outflowing gas compared to ammonia gas, it is possible to significantly increase (up to $2600 \mathrm{~N} \cdot \mathrm{s} / \mathrm{kg}$ ) the specific thrust impulse of the electro thermal engine [3].

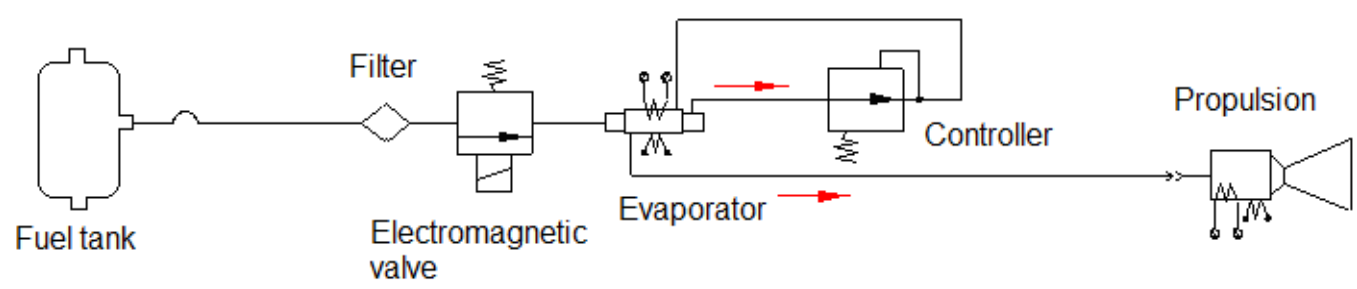

Figure 5. Schematic view of propulsion system.

For the micro-propulsion systems under consideration, there are no analytical models that allow determining the technical condition of the object, fuel consumption and its reserves with the necessary accuracy. At the same time, in the process of designing and testing, a large amount of computational and experimental information is accumulated, which can be used to create a predictive model of a micro-propulsion system based on neural networks technologies.

In the process of control the fuel consumption data received telemetric information (table 1), parameters of the control program, including the settings of the operating modes of the micropropulsion system, data on the previous turn on of the micro-propulsion system are used (figure 6 , table 2).

Table 1. Data set of the microsatellite telemetric information.

\begin{tabular}{ccccccc}
\hline Time & $I_{b s}, \mathrm{~A}$ & $I_{a b 1}, \mathrm{~A}$ & TxVAP & ON12 & $U_{b s}, \mathrm{~V}$ & $U_{a b 1}, \mathrm{~V}$ \\
\hline 13:03:04 & 0.59 & 1.33 & VAP1 & 0 & 13.8 & 13.9 \\
13:02:04 & 0.69 & 1.17 & VAP1 & 0 & 13.6 & 13.8 \\
13:01:04 & 0.53 & 1.38 & VAP1 & 0 & 13.6 & 13.7 \\
13:00:04 & 0.43 & 1.22 & VAP1 & 0 & 13.4 & 13.4 \\
$12: 59: 04$ & 1.17 & -0.59 & VAP1 & 0 & 12.1 & 12.2 \\
$12: 58: 04$ & 0.64 & -0.32 & VAP1 & 0 & 12.2 & 12.4 \\
$12: 57: 04$ & 0.85 & -0.43 & VAP1 & 0 & 12.2 & 12.3 \\
$12: 56: 04$ & 0.64 & -0.32 & VAP1 & 0 & 12.2 & 12.4 \\
$12: 55: 04$ & 0.85 & -0.43 & VAP1 & 0 & 12.2 & 12.3 \\
$12: 54: 04$ & 0.85 & -0.43 & VAP1 & 0 & 12.2 & 12.3 \\
$12: 53: 04$ & 0.69 & -0.38 & VAP1 & 0 & 12.2 & 12.4 \\
$12: 52: 04$ & 1.07 & -0.54 & VAP1 & 0 & 12.1 & 12.2 \\
$12: 51: 04$ & 0.64 & -0.32 & VAP1 & 0 & 12.2 & 12.4 \\
$12: 50: 04$ & 0.85 & -0.43 & VAP1 & 0 & 12.1 & 12.3 \\
$12: 49: 04$ & 0.75 & -0.38 & VAP1 & 0 & 12.2 & 12.4 \\
$12: 48: 04$ & 0.96 & -0.48 & VAP1 & 0 & 12.1 & 12.3 \\
\hline
\end{tabular}




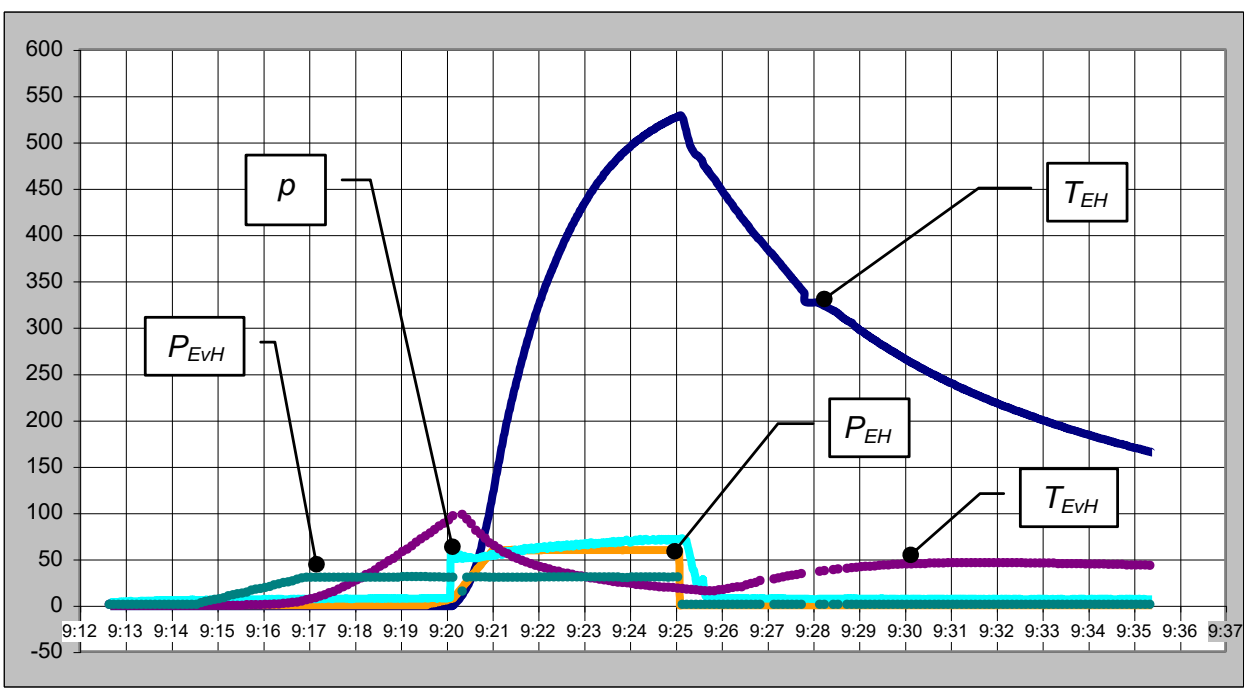

Figure 6. Data set of microsatellite parameters state.

All data that we have received on the different stages of the life cycle are stored in the information life cycle support system. This system includes the high performance computers, data warehouse subsystems, predictive analytics software and etc.

During operation, such a predictive model will allow solving the following tasks:

- evaluate the fuel reserves on board the microsatellite;

- diagnose the technical condition of the micro-propulsion system and detect its failures;

- determine the fuel consumption required to complete the orbital maneuver.

\section{Predictive neural network of the fuel consumption model}

In the process of modeling, telemetric information, parameters of the control program, including the settings of the operating modes of the propulsion system, data on previous inclusions of the micro jet propulsion are used. A fragment of the list of used parameters and their description are presented in table 2.

Table 2. Description of the parameters.

\begin{tabular}{ll}
\hline Parameters & Description \\
\hline$T_{\mathrm{EH}}$ & engine heater temperature \\
$U_{\mathrm{EH}}$ & voltage on the engine heater \\
$I_{\mathrm{EH}}$ & engine heater current \\
$T_{\mathrm{EvH}}$ & evaporator heater temperature \\
$U_{\mathrm{EvH}}$ & voltage on the evaporator heater \\
$I_{\mathrm{EH}}$ & evaporator heater current \\
$N$ & number of the received block with initial data (settings) \\
$t_{\mathrm{N}}$ & instrument time value \\
$p$ & pressure in the engine chamber \\
$T_{\operatorname{maxEH}}$ & maximum engine heater temperature \\
$T_{\operatorname{maxEvH}}$ & evaporator heater maximum temperature \\
$P_{\operatorname{maxEH}}$ & maximum power of the electric current supplied to the engine heater \\
$P_{\mathrm{maxEvH}}$ & maximum power of the electric current supplied to the evaporator heater \\
$t_{\mathrm{EP}}$ & engine preparation time \\
$t_{\Sigma}$ & engine running time \\
\hline
\end{tabular}


The second group is the output data group $Y=\left\{T_{\mathrm{EH}}, T_{\mathrm{EvH}}, p\right\}, Y \in R^{1 \times 3}$. The input data are fed to the propulsion system to form a thrust impulse, while the output data allow the trust impulse to be estimated.

All developed neural networks are multilayer perceptrons trained in the backpropagation method. As a training sample, the calculated and experimental data obtained during the design and testing stages of the propulsion system were used. The structure of neural networks is given in table 3 .

Table 3. Neural Network Architecture.

\begin{tabular}{ll}
\hline NN & Neural Network Architecture \\
\hline NN1 & $12-5-3-3$ \\
NN2 & $3-3-5-12$ \\
NN3 & $15-6-4-2$ \\
\hline
\end{tabular}

The predictive neural network of the fuel consumption of the electrothermal micro propulsion engine includes three separate neural networks NN1-NN3, which are having multilayer perceptron structure trained with back propagation learning algorithm. System of neural networks NN1-NN3 and their architectures, logics block selecting simulation and monitoring modes are presented on figure 7.

The NN1 neural network is designed to determine the dependence of the output information $Y$ on the input $X$ and is used in the simulation mode of the propulsion system. The NN2 neural network solves the inverse problem and determines the dependence of the input information $\mathrm{X}$ on the output $\mathrm{Y}$. The results of the NN2 neural network are used in the monitoring mode to solve the problem of diagnosing the technical condition of the engine. The NN3 neural network is used to determine the fuel consumption $G_{\tau}$ and assess the technical state of $S_{\tau}$. In the NN3, data streams $X$ and $Y$ are used as input. Data on fuel consumption obtained in the process of testing the engine, can improve the accuracy and reliability of the training set. The complex of neural networks based on NN1, NN2 and NN3 is shown in figure 7.

In the simulation mode of the propulsion system, the logic unit provides interaction between the $\mathrm{NN} 1$ and the NN3, and in the monitoring mode, the NN2 and the NN3, which improves the accuracy of the fuel consumption estimate using additional information about the technical condition of the engine.

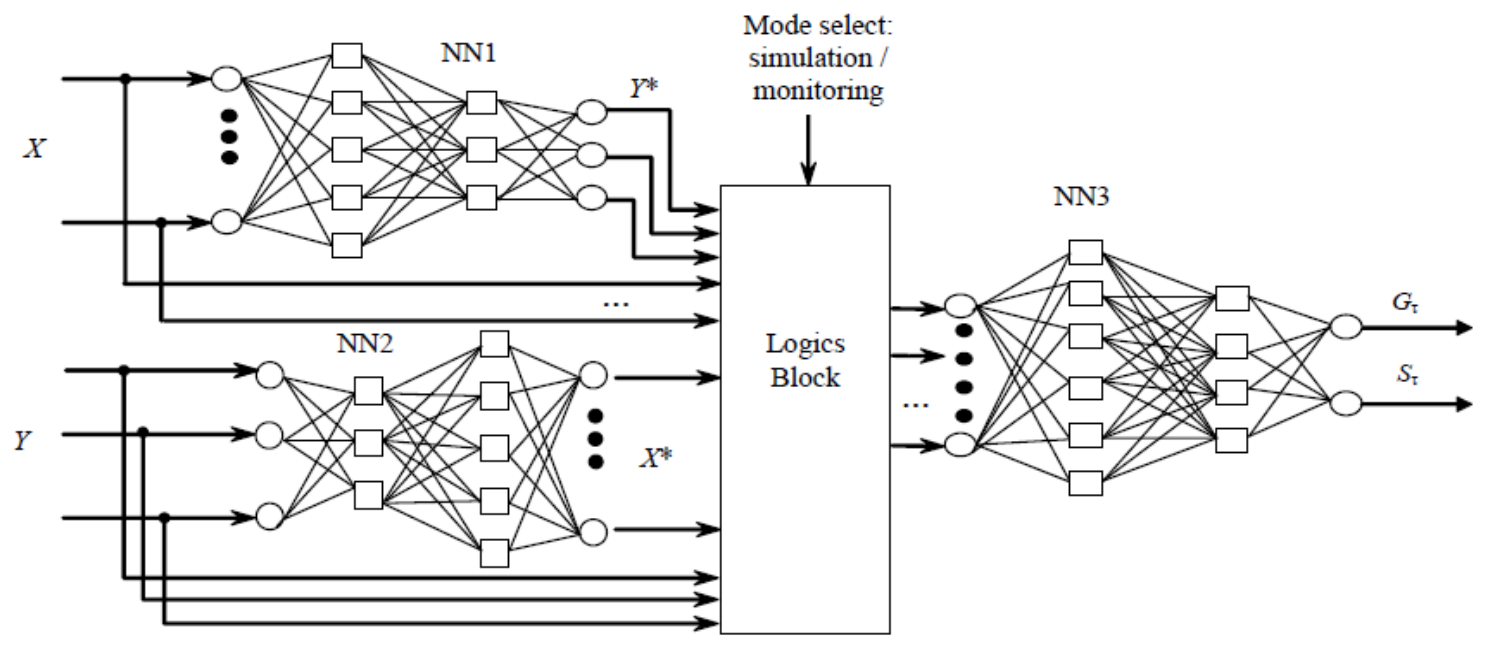

Figure 7. Predictive estimation of remaining fuel for microsatellite.

Data sets from databases of simulations results, telemetry information and control data, settings and additional information are applied for training processes for this system of neural networks. 

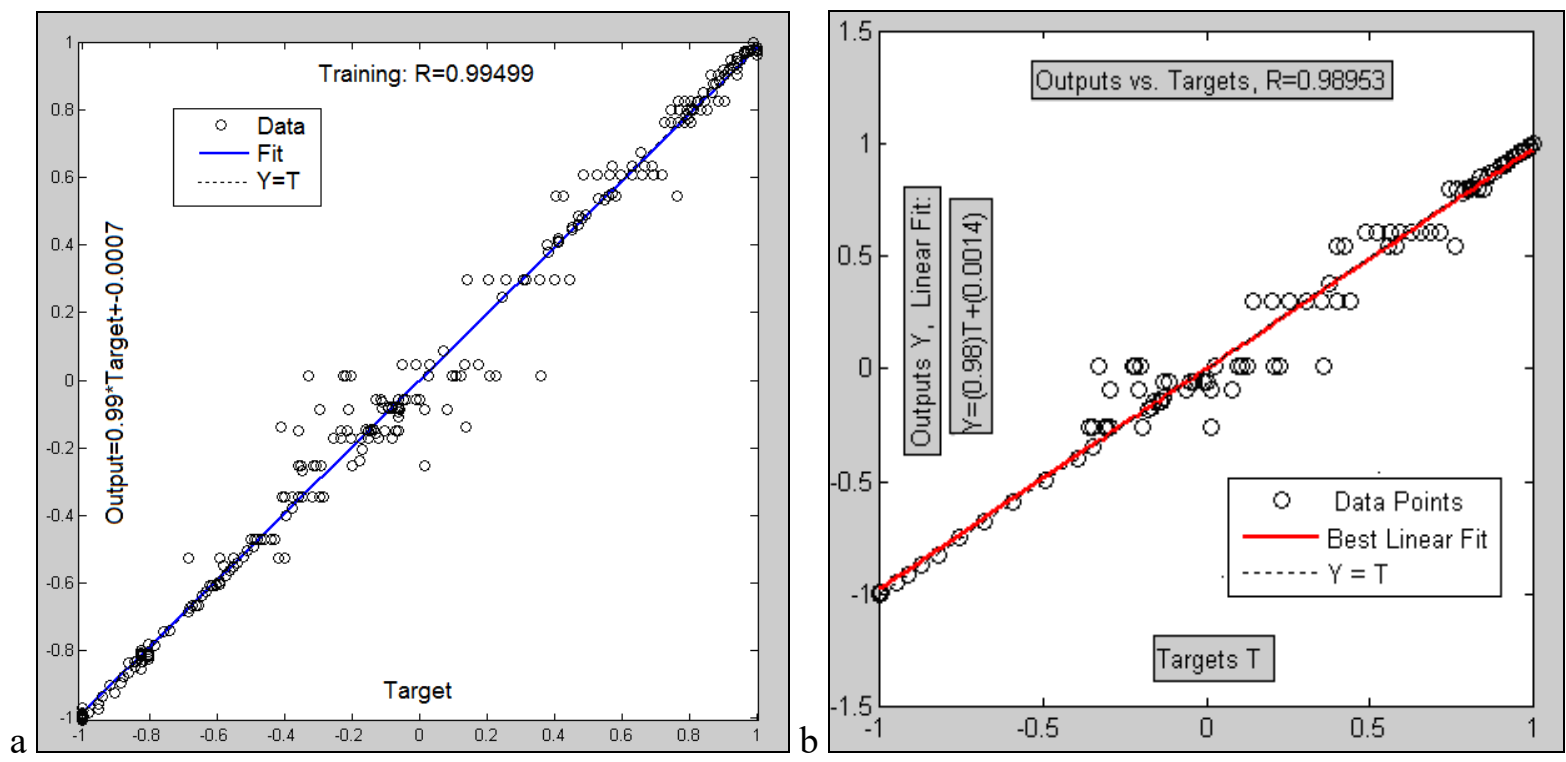

Figure 8. The predictive neural network model NN1 (a) and NN2 (b) learning results.

The telemetric information of a microsatellite with an electrothermal engine, which performs a single-pulse maneuver for orbit correction, is used as a training sample. Data sets include telemetry information on temperature of fuel thermal condition, fuel pressure, and control unit state. The learning results of the NN1 neural network are presented in figure 8. As we can see, the quality of training of the neural network is good. The architecture of NN1 neural network includes the input layer, hidden layer and output layer and has the following structure 12-5-3-3 neurons. To assess the reliability of the results of the neural network, a regression analysis was performed, obtained by comparing the reference values with the results of processing a test sample. For this, the "postreg" function is used in the MATLAB environment. The correlation coefficient between output prediction and sample data is $\mathrm{R} \approx 0.995$ (figure 8 , a). The result of the regression analysis for the neural network $\mathrm{NN} 2$ is presented in figure $8, \mathrm{~b}$ ). The correlation coefficient is $\mathrm{R} \approx 0.989$. The architecture of NN2 neural network includes the input layer, hidden layer and output layer and has the following structure 3-3-5-12 neurons. The experiment on teaching the intellectual model and determining the fuel supply showed that the accuracy of solving the problem under consideration increased by $10 \%$ compared with the analytical method.

\section{Conclusion}

One of the modern ways to achieve efficiency of the life cycle of complex technical systems is application of predictive analytics information support in design, production and exploitation stages of the life cycle. As it known the life cycle of the complex technical objects usually contains the following stages: prototyping, designing, manufacturing, implementation, operation, utilization. The duration of stages from prototyping to implementation for microsatellite is more times longer the duration of exploitation stage. One of the modern ways to achieve efficiency of the life cycle of such systems is application of predictive analytics information support in design, production and exploitation.

Predictive models and methods for the life cycle information support on the basis of the system of neural networks are proposed. The system of neural networks that can be estimated on onboard computer is presented. This predictive model can solve the task of estimation of fuel consumption, diagnostics and possible failures detection. 
The initial data for training neural networks are formed on the basis of the results of simulation modeling and telemetry data. The input values are normalized relative to the maximum values of these quantities. The initial weights of neural networks are set randomly.

The research results are expected to be used in the development of predictive analytics systems for small satellites in the future.

The proposed development technique can be used in building models for other types of engines of this class. And in the event of a change in parameters, it is necessary to develop a modified architecture of the neural network model.

\section{References}

[1] Kropotov Y A, Proskuryakov A Y and Belov A A 2018 Method for forecasting changes in time series parameters in digital information management systems Computer Optics 42(6) 1093-1100 DOI: 10.18287/2412-6179-2018-42-6-1093-1100

[2] Mostovoi J A 2012 Simulation mathematical model of the external ambience in life cycle of onboard software of management cosmic platform Computer Optics 36(3) 412-418

[3] Sweeting M N 2018 Modern Small Satellites-Changing the Economics of Space Proceedings of the IEEE 106(3) 343-361

[4] Blinov V N 2015 Design features and research of Electrothermal Microthrusters with Autonomous Heating Elements for the Purposes of Small Space Vehicle Orbital Manoeuvring Indian Journal of Science and Technology 8(27) IPL0581

[5] Blinov V N 2015 The studies of Small Space Vehicles Ammoniac Electrothermal Engine Units Design and Structural Layout Modern Applied Science 9(5)

[6] Yan Z 2013 System Dynamic Characteristic Simulation of Spacecraft Propulsion System Based on AMESim Advanced Materials Research 605-607 679-683

[7] Carroll D L, Cardin J M, Burton R L and Benavides G F 2015 Propulsion unit for CUBESATS (PUC) 62nd JANNAF Propulsion Meeting (7th Spacecraft Propulsion) URL: http://www.cuaerospace.com/Portals/SiteContent/Publications/2015/Carroll_JANNAF_ 2015_PUC_4059.pdf

[8] Zelinka I, Chen G, Rössler O E, Snásel V and Abraham A 2013 Nostradamus 2013: Prediction, Modeling and Analysis of Complex Systems Nostradamus conference Advances in Intelligent Systems and Computing (Ostrava Czech Republic Springer)

[9] Kondratyeva N, Valeev S 2016 Fatigue test optimization for complex technical system on the basis of lifecycle modeling and big data concept Proceedings of $10^{\text {th }}$ conference on Application of Information and Communication Technologies AICT 1-4

[10] Vasilyev V I, Valeyev S S and Shilonosov A A 2001 Design of neurocontroller for gas-turbine engine multi-mode control Proc. 8th Int. Conf. on Neural Information Processing ICONIP

[11] Kondratyeva N V, Valeev S S 2016 Simulation of the life cycle of a complex technical object within the concept of Big Data CEUR Workshop Proceedings 2004 216-223

[12] Karimov R R, Islamgulov T V and Karpenko O Yu 2012 Application of neural network and genetic algorithms to life cycle information support of complex technical object Proc. of 14th Int. Workshop on Computer Science and Information Technologies 1 228-231 